\title{
Mathematical Modelling and Simulation of Throughput in a Robotics Manufacturing System
}

\author{
Ganiyat Salawu ${ }^{1}$, Glen Bright ${ }^{2} \&$ Chiemela Onunka $^{3}$ \\ ${ }^{l}$ Department of Mechanical Engineering, University of KwaZulu-Natal, Durban, South Africa. \\ ${ }^{2}$ Department of Mechanical Engineering, University of KwaZulu-Natal, Durban, South Africa. \\ ${ }^{3}$ Department of Mechanical Engineering, Mangosuthu University of Technology
}

ORCID 0000-0002-7436-6721 (Ganiyat Salawu)

\begin{abstract}
The paper presented the impact of robots in a pick and place task in an advanced manufacturing environment. The study seeks to address the problem faced by manufacturers trying to obtain optimal throughput when a robot is available to perform a difficult task. Classical models with assumed parameters were used to describe a scenario of a pick and place task in a virtual manufacturing environment. The models were studied and analyzed using Queuing theory and the renewal reward theorem. Various expressions were developed, and the engineering equation solver (EES) was used for the numerical computation. The numerical results were simulated using MATLAB to achieve the desired results and illustrated graphically. An equation that can be used to calculate optimal throughput was derived. The outcome of study suggested a model that can be effective when solution to optimal throughput is required during the pick and place task of a robotic line manufacturing system.
\end{abstract}

Keywords: Advanced Manufacturing, Modelling, Optimal throughput, Robots, Simulation.

\section{INTRODUCTION}

Mathematical modelling and simulation have been a useful tool during the decision-making stage of manufacturing to predict the long run prospect of a manufacturing environment. Mathematical modelling is essential to study the best process and parameters that can be implemented to yield optimal productivity in an advanced manufacturing environment. Effective optimization usually makes use of sequences of automation to improve product quality, reduce time spent, and to increase throughput in an industrial system [1]. The use of robotic as a disruptive technology to replace human labor has been efficient, reliable, and highly flexible when performing repetitive task in an advanced manufacturing environment [2] [3]. The fourth industrial revolution has led to the transformation of the complex traditional manufacturing system into a flexible autonomous system. The fast growth in the revolution also led to the replacement of human labour with high-speed automation tool and machinery. The use of automation tool like robotic manufacturing system ease task of manufacturers when high precision and repetitive task are required [4]. Increase in the use of automation in manufacturing environment to perform a complex task has led to the development of various types of robot suitable in an advanced manufacturing environment. Fanuc robot has been effective in a manufacturing environment because of its explicit design characteristics. Fanuc robot is designed with a high degree of accuracy and capable of performing multiple manufacturing task. A typical Fanuc robot is shown in Fig. 1. The Fanuc robot was developed with a suitable software that is programmable to coordinate pick and place task of many parts within a short period of time. A vision camera integrated along with its operating system senses arriving parts and promptly send a signal to a robot for immediate detection to pick up as programmed. The use of a robot to automate a task in an advanced manufacturing environment, saves production time, production cost, and also assists manufacturers to achieve increased throughput.

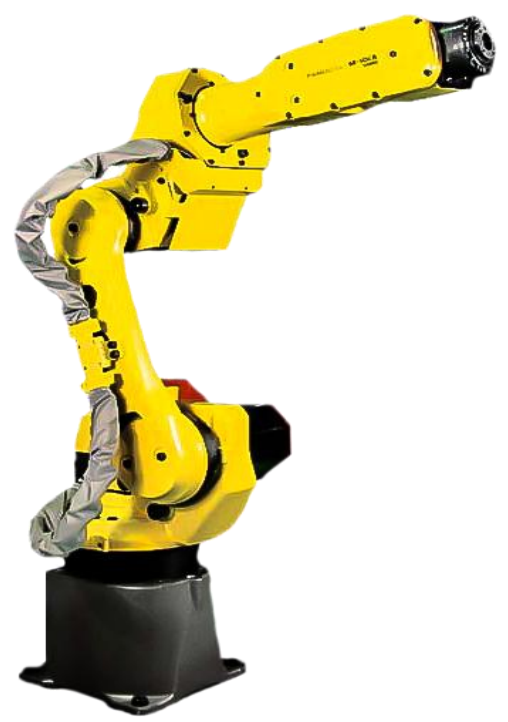

Figure 1. A typical Fanuc robot for a pick and place task 
In this present study, a single server robotic system with part arriving from various stations for a pick and place task was studied using mathematical models. Assumptions were made to represent the virtual manufacturing scenario. Classical mathematical models were used to describe various stages involved in the virtual manufacturing system. Using the queuing Markovian theory, and the renewal reward theorem, an expression was derived for estimating the system throughput. The Engineering equation solver (EES) was used for the numerical analysis, and MATLAB software was used as the simulation tool to derive optimal results. Results obtained were further illustrated graphically and discussed.

\section{RELATED WORK}

Previous studies have showed how robots have been used in manufacturing environment to improve manufacturing productivity. Some scholars applied disruptive techniques using different methods such as modelling, simulation and optimization to study manufacturing related problems and solutions were also suggested using various approach. For example, optimization method has been studied and used by a number of researchers. Troels used the simulation-based approach during the optimization study of an adaptive robotic manufacturing system that perform a pick and place task on deformed part [5]. The effectiveness of the scanner was improved by integrating a lightening system to a robotic system to perform a pick and place task. This proposed method was able to improve the robotic system performance for higher throughput. In another research, numerical optimization approach was developed to study and analyzed a suitable process that can facilitate a robotic handling of delicate manufactured parts.

An improved technology was devised that can quickly detect various shapes arriving from a conveyor belt in an advanced manufacturing environment [6]. The new technology has been efficient to increase throughput of the manufacturing station. The research presented in [7] [8] [9], shows how robotic manufacturing system can be optimized for optimal efficiency and productivity. The positioning of the part to be picked up is an area that affects robotic throughput in an advanced manufacturing environment. With adequate selection and with the simulation-based optimization approach, proper solution that can improve throughput during robotic machining system was developed by considering methods to adjust work pose for easy machining [8] [10].

Similarly, another robotic problem was optimized by effectively maximizing the working speed of a bipedal robotic system. The optimization process based on the approach of function fitting was used to predict the best parameters that can produce the fastest robot [11]. Also, in another research, the optimization of operation of the chicken fillet harvesting process was achieved by designing a system that has a virtual camera, robotic arm and gripper to separate the fillet. The automated system developed was capable of separating chicken fillet from the chicken carcass effectively [12]. The use of queuing modelling theory has seen to be effective for model analysis to describe how the virtual manufacturing system would behave in real-life manufacturing scenario. Different approaches using numerical approaches have been studied and implemented into queuing theory to study performance of manufacturing system mathematical models. The queuing mathematical theory was used effectively to describe the behavior of a mathematical model designed for inventory purpose. The queuing theory was effective for the system flexibility with improved productivity [13].

In the work of Anupana and Solankin [14], queuing system with unreliable server experiencing breakdown was analyzed. The Laplace transformation was applied for adequate measure of system performance. Numerical method was used to present outcome of the analysis [14]. Similarly, in another research, a queuing system with an unreliable server was also considered whereby, a backup server was assumed to take up action in case of server breakdown. Matrix geometric technique was used to determine some useful performance measures for the manufacturing line. The derivation extended to the derivation of Laplace- transform which was employed to illustrate the system performance numerically [15]. Furthermore, the Markovian queuing theory was implemented during the decision-making stage of a multi-stage production system [16]. The queueing theory was used to study the machine parameters that could yield an optimal productivity. This was further analyzed under the Bernoulli's condition to obtain their desired results [16].

The behavior of arriving part from a buffer station is also an area of interest to the present researchers. This can provide adequate knowledge of how to design a functional model that can be useful when making decision during an earlier stage of manufacturing. On this basis, various research has been studied. A single-server with discouraged arrival of customers, having retention of reneging customers was considered for study by Kumar and Sharma [17]. Models were developed to describe the system, whereby the Runge-Kutta method was used alongside with the queuing theory to analyze model performance. The MATLAB software was used for numerical computation of their results [17]. In another research by Kumar and Sharma, a steady state equation was derived to find solution to a Markovian single server queuing system having discourage arriving customers. Iteration method was implemented to solve the steady state equation using parameters used during the description of the model in their study [18].

Reliability of manufacturing system is also a contributing factor that has great impact on production throughput. Reliability test is essential in the early stage of the manufacturing process when considering the equipment life cycle and failure rate. Proper prediction of equipment reliability assists manufacturers to have a clear understanding of potential degradation that could occur during service and to proffer adequate solution when required. The description of reliability of a manufacturing system that uses conveyor and robotics in its application has been studied in [19]. The manufacturing system was analyzed to study the system reliability, and availability of unexpected failure along robotic manufacturing line [19].

Another research suggests that desirable allocation of buffer can also enhance production throughput in an advanced manufacturing environment [20]. Furthermore, an attempt to 
control congestion from arriving customers from joining existing queue was evaluated by Abdul Rasheed and Monoharan [21]. Service switches was introduced into the discouraged arrival of the Markovian queuing system under study. MATLAB was also used for analyzing the process for the best optimization parameter [21]. Most of the past research have only laid emphasis on system performance.

Only few attempted to optimize throughput by taking into consideration of the design parameters of machineries. In this present study, the modelling stage suggests parameters for the moving conveyor and these parameters were analyzed to study manufacturing environment. its effectiveness in an advanced manufacturing environment. The engineering equation solver (EES) that solves mathematical equations accurately was used and simulation were also carried out within a range of values using MATLAB software to achieve optimal result.

\section{METHOD}

The flow chart in Fig. 2, gives the steps involved during the modelling stage of a pick and place task in a virtual

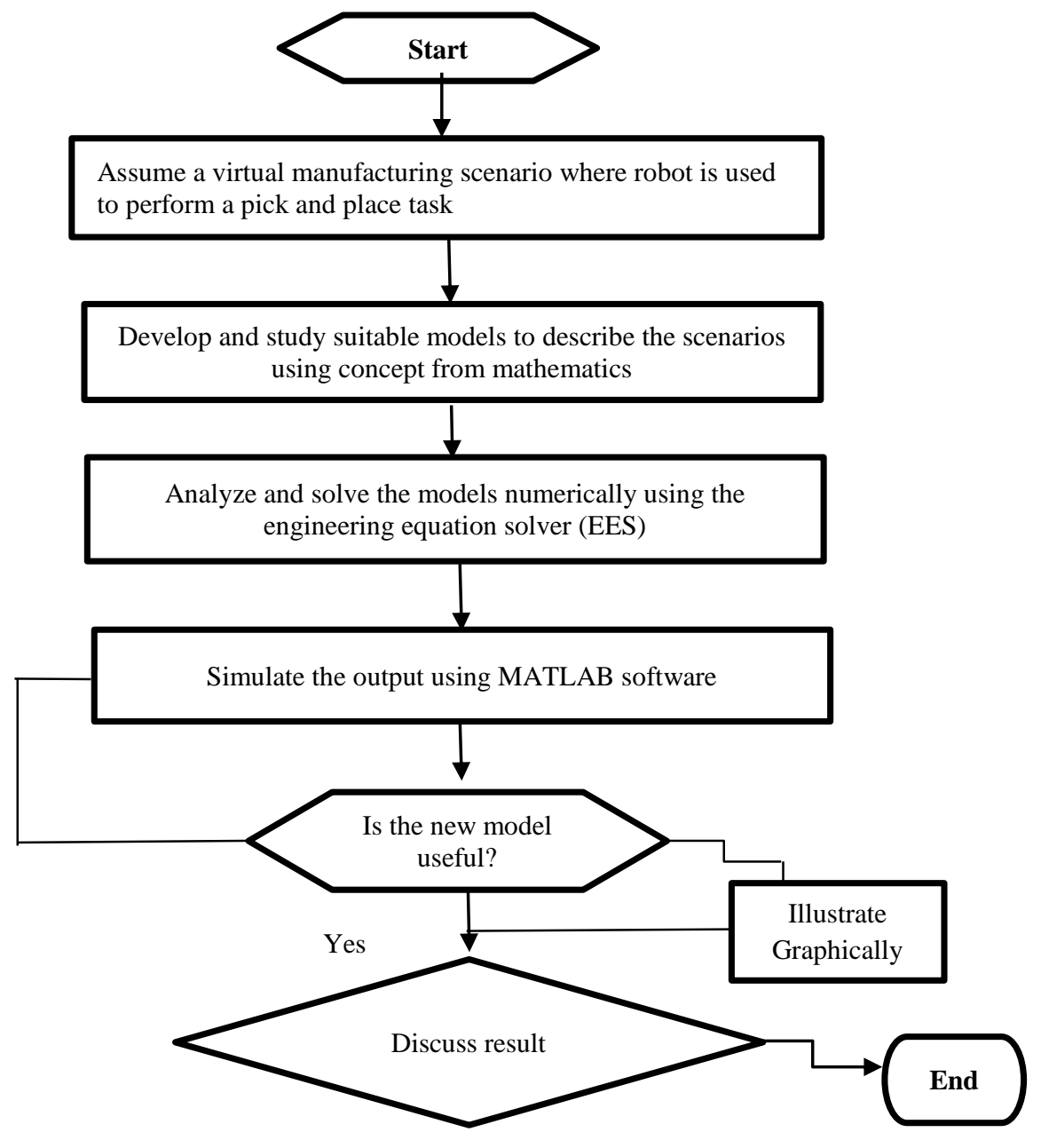

Figure 2: Flow chart for the proposed model

The system model considers a single server queue where parts arrives via conveyor belt from multiple station to a buffer station. The arriving parts join another conveyor where parts were transported to be picked up and placed by a robot in a virtual manufacturing environment. The arrival behavior is in a random manner following a Poisson process with varying with mean arrival rate $\lambda$, which is a case of inter arrival time (difference between two consecutive arrivals). The arrival of the parts from buffer station takes the form of a negative exponential distribution which follows the manner of impatient behavior of customers in the M/G/I queuing system.
The repeatability motion of the robotic arm makes the system to experience a deterministic feeding time $\mu$ (mean service time). Parts that were not picked up decides to leave and return back for service in the next cycle. This behavior in queuing theory is also referred to as reneging behavior of customers.

Consequently, the total cycle time $t_{c}$ required for the robot to complete a cycle was calculated using the total duration that was associated with the pick and place operation. In this model, it was assumed that the conveyor was not staved nor saturated. Following the inter-arrival behaviour, the tolal cycle time $t_{c}$ 
was obtained by summing together the time involved in the manufacturing scenario that was studied. Given that $t_{m}$ is the time at which parts move pass the conveyor, $t_{v i s}$ represent the camera vision time, $t_{p}$ as the pick and place time, $t_{t}$ is the time taken to change tool, and $t_{r}$ equals the time taken by the unpicked parts to re-circulate and join the next queue. The total cycle time was calculated using equation (1).

\section{III.I EQUATIONS AND ANALYSIS}

$$
t_{c}(s)=t_{m}+t_{v i s}+t_{p p}+t_{r}+t_{t}
$$

Assuming $t_{m}=0.13 s, t_{v i s}=0.1 s, t_{p}=0.12 s, t_{r}=0.125 s, t_{t}=0.15 s$.

With the notations and parameters presented below, a model that can describes the behavior of the pick and place scenario was presented.

\section{Parameters}

$\lambda=$ The mean arrival rate of parts from machines.

$\mu=$ The mean service rate of product also called inter - arrival time.

$p_{n}=$ probability of having $n$ part in queue.

$\rho=$ utilization or traffic intensity.

$L_{q}=$ Average number of parts in the queue

$L=$ Average number of parts in the system

$w_{q}=$ Average waiting time of parts on a conveyor before being served

$w_{a}=$ Average time a part spent in the whole system

$t_{c}=$ Total cycle arrival time

$t_{\text {vis }}=$ time taken by a camera to visualize part and send signal to robot

$t_{p}=$ time taken by the robot to feed and pick a part

Using the above notations from the queuing mathematical theory, a single server system was considered whereby the arriving parts are served by a single robot which identifies and pick up required part. Let the probability function of negative exponential distribution with inter-arrival time $\mu$, having a mean number of arrivals of parts from available stations be . The probability Pr that one product arrive at a time $(\mathrm{t})$ is given as $\lambda x=(\mathrm{t}, \mathrm{t}+\mathrm{x})$ for $\mathrm{x} \rightarrow 0$. Similarly, the probability Pr that more than one part arrive is given as $\rho_{n}\{(t+x), \mathrm{x} \rightarrow 0\}$. Let $x(t)$ at $t \geq 0$ denote the available product present to be served at a given time $t$.

Therefore, the system behavior can be represented mathematically using a probability function

$$
f(x)=\frac{e^{-\lambda} \lambda x}{x !}
$$

$\rho_{n}(t)=[n$ part arrival at a specific time $(0, t)]$

With the set of asumption presented, $\rho_{n}(t+x), \mathrm{x} \rightarrow 0$, can be expressed as

$\rho_{n}(t+x)=\rho_{n}(t)[1-\lambda x]+\rho_{n-1}(t) \lambda x$

The probability above represent the poisson arrival condition of parts.

$\rho_{0}(t+x)=\rho_{0}(t)[1-\lambda x]$

This can also be expressed using $\mathrm{h}$ to denote the inter- arrival time 
$\frac{\rho_{n}(t+h)-\rho_{n}(t)}{h}=-\lambda \rho_{n}(t)-\lambda \rho_{n-1}(t)$

Similrly, $\frac{\rho_{0}(t+h)-\rho_{0}(t)}{h}=-\lambda \rho_{0}(t)$

Diferentiation of $5 \& 6$ gives

$\frac{d \rho_{n}(t)}{d t}=-\lambda p_{n}(t)+\lambda \rho_{n-1}(t)$

$\frac{d \rho_{0}(t)}{d t}=-\lambda \rho_{0}(t)$

Equation 7 and 8 gives the set of the differential equation solving with respect to

$\rho_{0}(t)=e^{-\lambda t}$

To proof the above, the derivative of $\rho_{0}(t)$ is computed and compared with equation $7 \& 8$ to obtain $\rho_{1}(t)$,

$\rho_{1}(t)=\lambda t e^{-\lambda t}$

Equation 7 can be written as

$\rho_{n}(t)=\frac{(\lambda t)^{n}}{n !} \times e^{-\lambda t}$, when $n=0,1,2,3,4 \ldots$

For an efficient productivity, $\rho=\frac{\text { product arrival rate }(\lambda)}{\text { service rate }(\mu)}<1$

For a discrete time, $\rho_{n}(t)=\frac{\text { mean arrival rate }}{\text { mean service rate }}=\frac{\lambda(t)}{\mu(t)}$

Equation 11 can be used to obtain the probability that parts have been cleared from the work envelope. This equation expresses the relationship between the arrival rate and the server rate which can be used to obtain the utility rate of server.

Similarly, using notations from Queuing System (M/G/1) with Single Server, the average number of parts in queue, averagge number of parts in the system, average time spent before served, and average time spent in the system is given in equation $13,14,15 \& 16$.

Average number of parts in the queue $L_{q}=\frac{\lambda^{2}}{\mu(\mu-\lambda)}$

Average number of parts in the system $L=L_{q}+\frac{\lambda}{\mu}$

Average time a part that waits before being served $w_{q}=\frac{L_{q}}{\lambda}$

Average time a part spent in the system $w=w_{q}+\frac{1}{\mu}$

To predict a production optimal throughput, the cost involve during the pick and place task must also be considered by taking into consideration the average time spent and average number of parts in the system.

Given that

Cost of server (robot) $=C_{s}$, Cost of parts wating before picked up $=C_{w}$,

Cost of busy robot $=C_{b}$, Cost of idle robot $=C_{i}$, Cost of part returning to be picked $=C_{r}$.

The expression given in equation 16 can be considered to arrive at a total cost for the process,

$C_{p}=C_{w} \times \mathrm{L}+C_{b} \times \mathrm{w}+C_{r} \times w_{q}+C_{i} \times L_{q}+C_{s} * S$ 
During the pick and place task, the robot does not pick up all arriving part as the conveyor move past the vision camera. Other parts parts were recycled to join the next arriving parts. During the travelling period of parts, there exists a continuous movement of part whereby a minimum distance/gap $\alpha$, exist between the work envelope and the clear part boundaries. For effective throughput computation, the distance between two arriving part must not be less than $\alpha$ for the robotic arm gripper to easily pick-up parts. Assuming the number of visible parts is fed along the work envelope as $N_{f}$, the center point of the arriving part must be known. This can be obtained by considering the fact that the height and width of work envelope is the same. The diameter of the part fed in this model was assumed to be less than $20 \mathrm{~mm}$. Assuming both belts has the same velocity, the throughput equation can be easily obtained with the set of parameters and assumption made.

\section{Assumptions}

System is not saturated or starved.

$>$ There exist continious motion of buffer belt

$>$ The width and height of work envelope is assumed to be equal

All part are well secured to avoid falling off

Using the following notations and working parameters, suitable equations were derived.

\section{Notation/parmeters}

$N_{f}=$ number of part fed through the work envelope

$c=$ expected number of cleared parts

$d=$ diameter of fed part

$a=$ minimun clearance required $=d / 2$

$w=$ Height and width of workpiece

$P_{b}=$ proability that a work has been cleared from

boundry of the work envelope

$v=$ velocity of belt

$T_{r}=$ throughput rate of robot

$r_{b}=$ rate of arrival of parts from conveyor to server in Parts $/ \mathrm{mm} 2$
The renewal theorem of impatient customer is implemented for deriving a suitale equation for optimal throughput. The velocity of the conveyor belt is asuumed to be the same and represented with $\mathrm{v}$, using equation 17 , the conveyor speed was determined. Where $D$ is the diameter of the motor pulley, and $n$ represent the number of revolutions per seconds.

$$
\begin{aligned}
& V=\frac{\pi D n}{60} \\
& p_{b}=\frac{(w-d-2 \alpha)^{2}}{(w-d)^{2}}
\end{aligned}
$$

Given the centre of the work envelope as c, to obtain the centre of work envelope, the width and height of the work envelope was assumed to be equal. The diameter of the conveyor belt with the speed (v) was considered in equation 19.

$$
c=\frac{w \pi(\alpha+d)^{2}}{(w-d)^{2} v}
$$

Similarly, there exist another arrival rate denoted as $r_{b}$ which represents the arrival of parts from coveyor to the server (robot). Equation 20 was used to calculate the value for $r_{b}$.

$$
r_{b}=\frac{1}{c} \frac{(w-d)^{2} v}{w \pi(\alpha+d)^{2}}
$$

Also, the number of parts fed through the work envelope can be obtained by considering the width and height of work envelope, the velocity of conveyor, the probability of cleared work from work envelope, centre of work envelope, and the arrival rate of parts from buffer station to the pick up point. This is illustrated in equation 21. Equation 22 can be used to obtain the number of arrivin parts fed through the conveyor to the robotic system.

$$
\left[N_{f}\right]=\left(\frac{w}{v}\right) r_{b} p_{b} e^{-r b c}
$$

The throughput rate can be obtained using equation (23).

$$
T_{r}=\frac{N_{f}}{\left[\left(\frac{W}{v}\right)+t_{p}+\left[N_{f}\right] t_{c}\right]}
$$

The Engineering equation solver (EES) was used to solve the equations numerically and the results of the analysis were simulated using MATLAB software to achieve the best set of parameters for the system. With the set of equations and parameters assumed, optimal throughput was derived for a pick and place task.

Assumed values; $d=14.4, W=250 \mathrm{~m}, V=256$ rev per minutes, $T_{c}=0.625, n=340$ rev per minutes,

$a=\frac{d}{2}=7.2 \mathrm{~mm}$, Lamda $\lambda=0.5$ parts per sec, $\mu=0.4, c_{w}=50, c_{b}=0.1, c_{r}=60, c_{i}=10, c_{s}=0.5, t_{m}=0.13 \mathrm{~s}$,

$t_{\text {vis }}=0.1 s, t_{p}=0.12 s, t_{r}=0.125 s, t_{t}=0.15$ s. from calculations, $t_{c}=0.625 \mathrm{secs}$ 
International Journal of Engineering Research and Technology. ISSN 0974-3154, Volume 13, Number 1 (2020), pp. 137-145

(C) International Research Publication House. https://dx.doi.org/10.37624/IJERT/13.1.2020.137-145

\section{FIGURES}

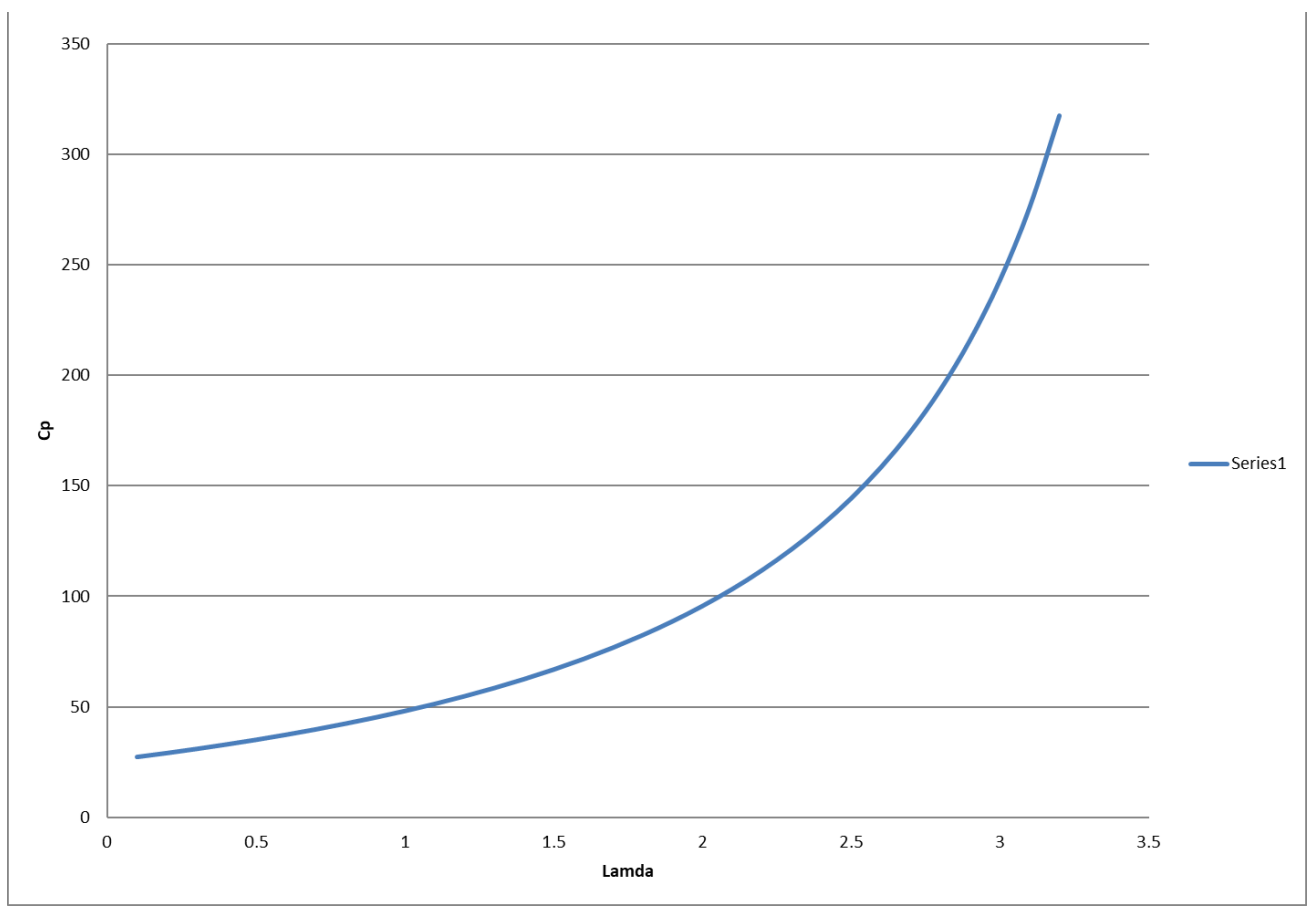

Figure 3: Cost of production against arrival rate

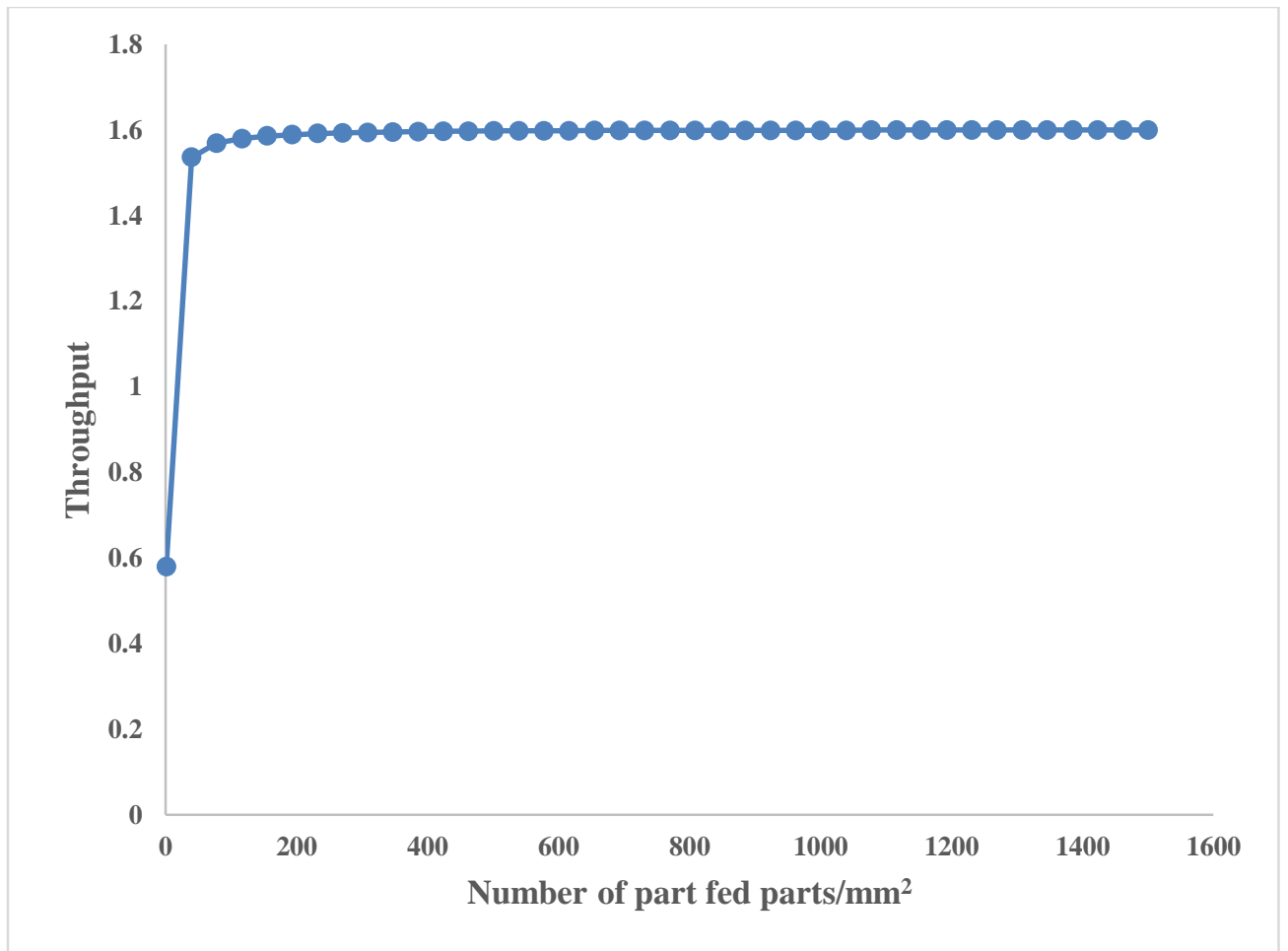

Figure 4: Graph of throughput against the number of parts fed through the conveyor

\section{DISCUSSION}

Considering the outcome from the analytical solution, we were able to arrive at an equation that can be used to obtain optimal throughput in a robotic manufacturing scenario. When computing the assumed parameters into the developed model, the graph of Fig. 3 illustrated the relationship between the mean interarrival rate and the cost of production. Outcome shows that the longer it takes the parts to arrive the robotic system the higher the cost of production. Also, the number of parts fed through the conveyor to the server (robot) was plotted against 
throughput rate in Fig. 4. The result shows that with the model and some assumed parameters used,an optimal throughput was obtained when the arrival rate was constant at 1.63 parts $/ \mathrm{mm}^{2}$. The results shows that the developed model can be used in the real life manufacturing system when optimal throughput is required to be obtained.

\section{CONCLUSION}

The purpose of the paper to derive a solution that could increse the throughput rate of a conveyor system during a pick and place task in a robotic manufacturing system. The research used claassical models to describe a virtual robotic manufacturng scenarios, whereby parts arriving from various stations were transported via a conveyor and a vision camera attached to a robot senses and send signal to the robots. The robots picks and place task in a random manner and other parts that were not picked up have to recirculate and join the next arriving parts. Considering the competitive manufacturing environment, the need to increase throughput to reduce cost of production is of highest interest among modern manufacturers. Various equations were derived and an equation for optimal throughput was developed. The equations were tested to determine the efficiency of the equations. With the set of assumed parameters, the engineering equation solver (EES) was used for the numerical analysis, and MATLAB was used to simulate the parameters against each other to obtain optimal outputs. From the classical models formulated, it can be concluded that the equation for optimal throghput was effective. The graphical illustration compares the mean arrival rate and the cost of production. Outcomes shows that the slower the arrival reate of parts, the higher the cost of production. Also, the grapg of throuhgput against arrival of part shows that in this study, an optimal throughput can be achieved at $1.6 \mathrm{parts} / \mathrm{mm}^{2}$. This model can be useful among manufacturers when optimal throuhput is required to be obtained in an advanced manufacturing environment.

\section{ACKNOWLEDGEMENTS}

The financial supports from the TETFUND Nigeria, is highly acknowledged. (TETFUND/DAST\$D/POLY/OFFA/ASTD/ 2017/VOL 1).

\section{REFERENCES}

[1] A. e. a. Brunete, "Hard Material Small-Batch Industrial Machining Robot.," Robot. Comput.-Integr. Manuf., vol. 54, p. 185-189, 2018.

[2] G. I. V, L. T.P, S. L, R. P and P. P., "Dynamic Characterization of Machining Robot and Stability Analysis.," Int. J. Adv. Manuf. Technol, vol. 82, p. 351359, 2016.

[3] A. Klimchik, A. Ambiehl, S. Garnier, B. Furet and A. Pashkevich, "Efficiency Evaluation of Robots in Machining Applications Using Industrial Performance
Measure," Robot. Comput.-Integr. Manuf, vol. 48, pp. 12-29, 2017.

[4] G. Grzegorz, K. Adrian and P. Iwona, "Modeling and Simulation of Manufacturing Line Improvement," International Journal of Computational Engineering Research (IJCER), vol. 6, no. 10, pp. 26-31, 2016.

[5] B. Troels, H. Sabastian, A. Henrik, W. Hiels and N. K, "An Adaptive Robotic System for Doing Pick and Place Operations with Deformable Objects," Journal of Intelligent and Robotic Systems, vol. 94, no. 1, pp. 81100, 2019.

[6] L. e. al, "An Adaptable Robot Vision System Performing Manipulation Actions With Flexible Objects," IEEE TRANSACTIONS ON AUTOMATION SCIENCE AND ENGINEERING, vol. 11, no. 3, pp. 749-765, 2014.

[7] S. Mousavi, V. Gagnol, B. Bouzgarrou and P. Ray, "Stability Optimization in Robotic Milling Through the Control of Functional Redundancies. Robot," Comput.Integr. Manuf, vol. 50, p. 181-192, 2017.

[8] Y. Tian, B. Wang, J. Liu, F. Chen, S. Yang, W. Wang and $\mathrm{L}$. $\mathrm{Li}$, "Research on layout and operational pose optimization of robot grinding system based on optimal stiffness performance.," J. Adv. Mech. Des. Syst. Manuf, vol. 11, 2017.

[9] L. Lin, H. Zhao and H. Ding, "Posture optimization methodology of $6 \mathrm{R}$ industrial robots for machining using performance evaluation indexes," Robot. Comput.Integr. Manuf, vol. 48, p. 59-72, 2017.

[10] Q. Haojie, L. Yuwen and X. Xiong, "Workpiece Pose Optimization for Milling with Flexible-Joint Robots to Improve Quasi-Static Performance," Applied Science, vol. 9, pp. 1-15, 2019.

[11] R. Calandra, A. Seyfarth, J. Peters, M. Deisenroth and Bayesian, "optimization for learning gaits under uncertainty," Ann. Math Artif. Intell, vol. 76, no. 1-2, pp. 5-23, 2016.

[12] Misimi et al., "GRIBBOT - Robotic 3D vision-guided harvesting of chicken fillets," Computer and electronics in engineering, vol. 120, pp. 81-100, 2016.

[13] S. M. Seyedhoseini, E. K. R. Reza and E. Zang, "Application of queuing theory in inventory systems with substitution flexibility," journal of Industrial Engineering International, vol. 11, no. 4, pp. 37-44, 2015.

[14] A. S. Anupama, "Mathematical Modeling and Analysis of Finite Queueing System with Unreliable Single Server," IOSR Journal of Mathematics (IOSR-JM), vol. 12, no. 3, pp. 8-14, 2016.

[15] D. Alexandra and V. Vladmir, "Analysis of an un-reliable single-server queue with back-up server," Communications in Computer and Information Science, vol. 499, pp. 149-161, 2015.

[16] S. Du, R. Xu and L. Li, "Modeling and Analysis of Multiproduct Multistage Manufacturing System for 
Quality Improvement," IEEE transactions on systems, man, and cybernetics: systems, vol. 48, no. 5, pp. 801820,2018

[17] R. \&. S. Kumar, "Time-Dependent Analysis of a SingleServer Queuing Model with Discouraged Arrivals and Retention of Reneging Customers," RT\&A, vol. 4, no. 47, pp. 84-90, 2017.

[18] R. \&. S. S. K. Kumar, " A single-server Markovian queuing system with discouraged arrivals and retention of reneged customers," Yugoslav Journal of Operations Research, vol. 24, pp. 119-216, 2014.

[19] A. Fudzin and M. Majid, "Reliability and availability analysis for robot subsystem in automotive assembly plant: a case study," Materials Science and Engineering, vol. 100, no. 1, pp. 1-7, 2015.

[20] H. Martirjn, A. Hadi, G. Marc, B. Twan, G. Ruben and D. S. V. Rob, "Monotonic Optimization of Dataflow Buffer Sizes," Journal of Signal Processing Systems, vol. 91, no. 1, pp. 21-32, 2019.

[21] K. \&. M. Abdul-Rasheed, "Markovian Queueing System with Discouraged Arrivals and Self-Regulatory Servers," Advances in Operations Research, vol. 2016, pp. 1-12, 2016.

[22] Fanuc, "Fanuc company," FANUC high industrial speed machine [Online] Available: http://www.fanuc.ed>robot>fanuc-m-10.a-125. [Accessed 29 August 2019].

[23] G. \&. K. G. Dadi, "Optimizing robotic part feeder throughput with queueing theory," Assembly Automation, vol. 27, no. 2, p. 134-140, 2007.

[24] "Robust optimization of robotic pick and place operations for deformable objects through simulation," in IEEE International Conference on Robotics and Automation (ICRA), Stockholm, Sweden, 2016.

[25] G. M. J. \&. M. J. D, "Mathematical Modeling of Production Scheduling Problem: A Case Study for Manufacturing Industry," International Journal of Science Technology \& Engineering , pp. 224-226, 2015. 